\title{
A GENERALIZATION OF ALTMAN'S ORDERING PRINCIPLE
}

\author{
MIHAI TURINICI
}

\begin{abstract}
A maximality principle on quasi-ordered quasi-metric spaces, containing, in particular, Altman's and the Brézis-Browder ordering principles, is given. As an application, a local mapping theorem extending - from a "functional" viewpoint - a similar one due to Altman, is derived.
\end{abstract}

Let $X$ be a nonempty set, and let $\leqslant$ be a quasi-ordering (that is, a reflexive and transitive relation) on $X$. Concerning existence of certain "maximal" elements in the structure $(X, \leqslant)$, the following Altman's ordering principle [3] must be considered as a starting point of our developments.

THEOREM 1. Let the quasi-ordered set $(X, \leqslant)$ be such that every descending sequence has a lower bound, and let $F: X^{2} \rightarrow R$ be a function satisfying $F(x, y) \leqslant 0$ when $x \leqslant y$, as well as:

(i) for any $y$ in $X, F(., y)$ is bounded below on $X(y, \geqslant)=\{t \in X ; y \geqslant t\}$;

(ii) for any $x$ in $X, F(x,$.$) is decreasing;$

(iii) $\limsup _{n \rightarrow \infty} F\left(x_{n+1}, x_{n}\right)=0$, for every descending sequence $\left(x_{n} ; n \in N\right)$ in $X$. Then, to any $x$ in $X$, there corresponds $z$ in $X$ with (a) $x \geqslant z$, and (b) $y \leqslant z$ implies $F(y, z)=0$.

As already pointed out by this author, Theorem 1 may be viewed as a natural and straightforward extension of the well-known Brézis-Browder ordering principle [4, 6], a result that found a number of interesting applications to convex as well as nonconvex analysis (see the above references); consequently, a generalization of it might be of some avail. It is precisely our main aim to formulate a statement of this kind; as a direct application, a generalization of a basic mapping theorem due to Altman [2] will be given.

Let $(X, \leqslant)$ be a quasi-ordered set. A function $d: X^{2} \rightarrow[0, \infty)$ will be termed $\leqslant-$ quasi-metric when $d(x, x)=0$ for all $x$ in $X$, and

(iv) for every $\varepsilon>0$ there exists $\delta>0$ such that $x \leqslant y \leqslant z$ and $d(x, y), d(x, z)<\delta$ imply $d(y, z)<\varepsilon$.

In such a context, a sequence $\left(x_{n} ; n \in N\right)$ in $X$ will be said to be $d$-asymptotic provided that $\liminf _{n \rightarrow \infty} d\left(x_{n}, x_{n+1}\right)=0$ and an element $z$ in $X$ will be termed $d$-maximal in case $z \leqslant y$ implies $d(z, y)=0$. Now, under these conventions, the main

Received by the editors December 8, 1982 and, revised form, April 4, 1983.

1980 Mathematics Subject Classification. Primary 54F05, 06A10; Secondary 39B70, 54C10.

Key words and phrases. Quasi-ordering, maximality principle, mapping theorem. 
result of the present note is

THEOREM 2. Suppose the structure $(X, \leqslant)$ satisfies

(v) any ascending sequence has an upper bound and let the $\leqslant$-quasi-metric $d$ on $X$ be such that

(vi) any ascending sequence is $d$-asymptotic.

Then, to every $x$ in $X$ there corresponds a d-maximal element $z$ in $X$ with $x \leqslant z$.

Proof. As a matter of fact, the proof of Theorem 2 is implicit in [7] but, for the sake of completeness, we shall, however, indicate its basic lines. Letting $x$ in $X$, it follows - by the same procedure as in the author's paper we already mentioned - that an ascending sequence $\left(x_{n} ; n \in N\right)$ in $X$ may be found with $x \leqslant x_{n}, n \in N$, and

$$
y \in X, n \in N, x_{n} \leqslant y \text { imply } d\left(x_{n}, y\right)<2^{-n} .
$$

By (v), $x_{n} \leqslant z, n \in N$, for some $z$ in $X$. Let $y$ in $X(z, \leqslant)=\{t \in X ; z \leqslant t\}$ and let $\varepsilon>0$ be arbitrarily fixed. There exists, by (1), an $n(\delta) \in N$ with $d\left(x_{n}, z\right)$, $d\left(x_{n}, y\right)<\delta, n \geqslant n(\delta)$ (where $\delta$ is that appearing in (iv)) and this gives $d(z, y)<\varepsilon$, ending the argument. Q.E.D.

Now, clearly, Altman's result follows from Theorem 2 if we replace $\leqslant$ by its dual and put $d(x, y)=|F(y, x)|, x, y \in X$ (note at this moment the boundedness hypothesis (i) may be dropped in the statement of Theorem 1, while (ii) may be rephrased as

(ii) $F(x, y) \geqslant F(x, z)+F(y, z)$ when $x \leqslant y \leqslant z)$.

On the other hand, in case $d$ is a quasi-metric (i.e., a nonsufficient metric) on $X$, Theorem 2 reduces to Theorem 1 of the above quoted author's paper, which turns out to be a generalization of the Brézis-Browder ordering principle distinct from that indicated by Altman. Moreover, calling the element $z$ in $X$, $\leqslant-$ maximal provided that $z \leqslant y$ implies $y \leqslant z$, it is evident that, under the supplementary hypothesis

(vii) $x \leqslant y$ and $d(x, y)=0$ imply $y \leqslant x$,

Theorem 2 yields a Zorn maximality principle in $(X, \leqslant)$ (for any $x$ in $X$ there exists a $\leqslant$-maximal element $z$ in $X$ with $x \leqslant z$ ). A more specific proof of this fact may be obtained along the following lines. Let $C$ be a $\leqslant$-chain in $X$; constructing as before an ascending sequence $\left(x_{n} ; n \in N\right)$ in $C$ satisfying (1) (modulo $C$ ) the following possibilities hold; either $\left(x_{n} ; n \in N\right)$ is cofinal in $C$ and then, any upper bound of it is clearly an upper bound of $C$, or $\left(x_{n} ; n \in N\right)$ is not cofinal in $C$ in which case, any upper bound (in $C$ ) of this sequence is, by (vii), an upper bound of $C$. So, any S-chain in $X$ is bounded above and the classical Zorn theorem applies. Of course, in case (vii) does not hold, Theorem 2 (hence, in particular, Altman's and/or the author's ordering principles we quoted before) cannot be viewed as a Zorn maximality principle in $(X, \leqslant)$. Finally, a slight generalization of our main result could be obtained if, instead of $d$, a denumerable family $D$ of $\leqslant$-quasi-metrics on $X$ were used. More specifically, assume (vi) is fulfilled for any $d$ in $D$; then, under the acceptance of (v), to every $x$ in $X$ there corresponds $z$ in $X$ with (a) $x \leqslant z$, and (b) $z \leqslant y$ implies $d(z, y)=0, d \in D$; an implicit proof of this fact may be found in [8].

Now, passing to the second part of our developments, let $(X, d, \leqslant)$ be a quasiordered metric space, and $(Y,\|\cdot\|)$ a normed space. Under the terminology of [7], let us call $\leqslant$ self-closed when the limit of any ascending convergent sequence is an 
upper bound of it, and the ambient space s-complete in case any ascending Cauchy sequence is a convergent one. Also, given the mapping $T$ from $D(T) \subset X$ into $Y$, let us term it $\leqslant$-closed provided, if the ascending sequence $\left(x_{n} ; n \in N\right)$ in $D(T)$ satisfies $x_{n} \rightarrow x$ and $T x_{n} \rightarrow y$ for some $x$ in $X$ and $y$ in $Y$, respectively, then $x \in D(T)$ and $T x=y$. Suppose in what follows, $X$ is a $\leqslant$-complete metric space under the self-closed quasi-ordering $\leqslant$, and $Y$ is a Banach space under its norm. As an interesting application of the main result, the following "local" mapping theorem can be stated and proved.

THEOREM 3. Suppose the $\leqslant$-closed mapping $T: D(T) \rightarrow Y$ and the element $x_{0}$ in $D(T)$ with $T x_{0} \neq 0$ are such that a number $q$ in $[0,1)$ and a couple of functions $b, f$ : $(0, \infty) \rightarrow[0, \infty)$ satisfying

$$
(1-s / t) b(t) \leqslant f(t)-f(s), \quad t, s>0, q t \leqslant s<t,
$$

may be found with the property: for any $x$ in $D_{0}(T)=\left\{y \in D(T) ; x_{0} \leqslant y\right.$, $\left.d\left(x_{0}, y\right) \leqslant f\left(\left\|T x_{0}\right\|\right) /(1-q)\right\}$ with $T x \neq 0$ there exist $x^{\prime} \geqslant x$ in $D(T)$ and $\varepsilon$ in $(0,1]$ with

$$
\left\|T x^{\prime}-(1-\varepsilon) T x\right\| \leqslant q \varepsilon\|T x\|, \quad d\left(x, x^{\prime}\right) \leqslant \varepsilon b(\|T x\|) .
$$

Then, the equation $T x=0$ has at least a solution in $D_{0}(T)$.

Proof. Suppose by contradiction that the assertion of the theorem were not valid; so, given any $x$ in $D_{0}(T)$ there exists $x^{\prime} \geqslant x$ in $D(T)$ such that (3) holds. Observe that, as an immediate consequence,

$$
\left\|T x^{\prime}\right\| \leqslant(1-(1-q) \varepsilon)\|T x\|, \quad\left\|T x-T x^{\prime}\right\| \leqslant r\left(\|T x\|-\left\|T x^{\prime}\right\|\right)
$$

where $r=(1+q) /(1-q)$. Putting $t=\|T x\|, s=(1-(1-q) \varepsilon) t$, we clearly have $q t \leqslant s<t$ so, by (2) plus (3) (the second part), we get (denoting $g(t)=f(t) /(1-q)$, $t>0)$

$$
d\left(x, x^{\prime}\right) \leqslant \varepsilon b(t) \leqslant(1-s / t) b(t) /(1-q) \leqslant g(t)-g(s) .
$$

Now, let $E_{0}(T)$ denote the subset of all $y$ in $D_{0}(T)$ with $d\left(x_{0}, y\right) \leqslant g\left(\left\|T x_{0}\right\|\right)-$ $g(\|T y\|)$ and observe that $x \in E_{0}(T)$ plus (4) implies $x_{0} \leqslant x^{\prime}$ and

$$
d\left(x_{0}, x^{\prime}\right) \leqslant g\left(\left\|T x_{0}\right\|\right)-g(s) \leqslant g\left(\left\|T x_{0}\right\|\right)
$$

whence $x^{\prime} \in D_{0}(T)$. It follows at once that $0<\left\|T x^{\prime}\right\| \leqslant s$ and therefore, by (4) again (observe that, as a consequence of (2), $g$ is increasing on its existence domain),

$$
d\left(x, x^{\prime}\right) \leqslant g(\|T x\|)-g\left(\left\|T x^{\prime}\right\|\right)
$$

proving $x^{\prime} \in E_{0}(T)$ too; moreover, as $T x \neq T x^{\prime}$, we necessarily have $x \neq x^{\prime}$. Let $e$ denote the "product" metric on $D(T)$,

$$
e(x, y)=\max (d(x, y),\|T x-T y\|), \quad x, y \in D(T),
$$

and let $\leqq$ indicate the ordering on $D_{0}(T)$ defined as

$x \leqq y$ if and only if $x \leqslant y, d(x, y) \leqslant g(\|T x\|)-g(\|T y\|)$ and

$\|T x-T y\| \leqslant r(\|T x\|-\|T y\|)$. 
Our main aim is to demonstrate that conditions (v)-(vii) are fulfilled in $\left(E_{0}(T), e\right.$, $\leqq$ ) and this will lead us to the desired contradiction. (Indeed, it would follow then by the main result that, for the given $x_{0}$ in $E_{0}(T)$, a $\leqq$-maximal element $z$ in $E_{0}(T)$ may be found with $x_{0} \leqq z$; on the other hand, by the above relations, a $z^{\prime} \in E_{0}(T)$ may be chosen so that $z \leqq z^{\prime}$ and $z \neq z^{\prime}$, showing $z$ is not maximal in $\left(E_{0}(T)\right.$, )). To this end, let $\left(x_{n} ; n \in N\right)$ be an ascending (modulo $\leqq$ ) sequence in $E_{0}(T)$, that is,

$$
\left\{\begin{array}{l}
x_{n} \leqslant x_{m}, \quad d\left(x_{n}, x_{m}\right) \leqslant g\left(\left\|T x_{n}\right\|\right)-g\left(\left\|T x_{m}\right\|\right) \text { and } \\
\left\|T x_{n}-T x_{m}\right\| \leqslant r\left(\left\|T x_{n}\right\|-\left\|T x_{m}\right\|\right), \quad n, m \in N, n \leqslant m .
\end{array}\right.
$$

As an easy consequence of this fact, $\left(g\left(\left\|T x_{n}\right\|\right) ; n \in N\right)$ and $\left(\left\|T x_{n}\right\| ; n \in N\right)$ are descending sequences in $[0, \infty)$ - hence Cauchy sequences-and therefore, $\left(x_{n}\right.$; $n \in N$ ) is an ascending (modulo $\leqslant$ ) $d$-Cauchy sequence in $X$ and $\left(T x_{n} ; n \in N\right)$ a Cauchy sequence in $Y$. By our completeness/closedness hypothesis, $x_{n} \stackrel{d}{\rightarrow} x$ and $T x_{n} \rightarrow T x$ for some $x$ in $D(T)$, proving $x_{n} \stackrel{e}{\rightarrow} x$ and establishing the e-asymptotic property (vi). Moreover, as $D_{0}(T)$ is closed with respect to ascending (modulo $\leqslant$ ) $d$-convergent sequences, it clearly follows that $x \in D_{0}(T)$ (hence $T x \neq 0$ ) in which situation, observing that, as a consequence of (5),

$$
d\left(x_{n}, x_{m}\right) \leqslant g\left(\left\|T x_{n}\right\|\right)-g(\|T x\|), \quad n, m \in N, n \leqslant m,
$$

and

$$
\left\|T x_{n}-T x_{m}\right\| \leqslant r\left(\left\|T x_{n}\right\|-\|T x\|\right), \quad n, m \in N, n \leqslant m,
$$

we immediately derive (performing the limit as $m$ tends to infinity) $x_{n} \leqq x, n \in N$ (and consequently, $x \in E_{0}(T)$ ) proving (v) and completing the argument, since (vii) is trivially satisfied in our case. Q.E.D.

Concerning the basic hypothesis (3), let $X$ be a Banach space and let $X_{+}$be a closed quasi-cone in $X$ (that is, $\lambda x+\mu y \in X_{+}$whenever $\lambda, \mu \geqslant 0$ and $x, y \in X_{+}$); then, indicating by $\leqslant$ the usual quasi-ordering induced by $X_{+}$, the following condition appears as a sufficient one for its validity:

(viii) for any $x$ in $D_{0}(T)$ with $T x \neq 0$ there exists $v$ in $X_{+}$with $d T_{x}(v)=-T x$ and $\|v\| \leqslant b(\|T x\|)$

(here, $d T_{x}$ indicates the usual Gâteaux derivative of the mapping $T$ at the point $x$ ); indeed, putting $x^{\prime}=x+\varepsilon v$, (3) becomes

$$
\left\|(T(x+\varepsilon v)-T x) / \varepsilon-d T_{x}(v)\right\| \leqslant q\|T x\|,
$$

a relation that clearly takes place for sufficiently small $\varepsilon$ in $(0,1]$. Note that such a condition will be fulfilled when, e.g., a $\delta>0$ may be found with

$$
-T x /\|T x\| \in d T_{x}\left(S(0, \delta) \cap X_{+}\right)
$$

for all $x$ in $D_{0}(T)$ with $T x \neq 0$ (where $S(0, \delta)$ denotes the closed sphere with center $0 \in X$ and radius $\delta$; see also Cramer and Ray [5]). On the other hand, a sufficient condition (of a practical interest) for (2) is

(ix) $t \vdash b(t)$ is increasing and $c(t)=\int_{0}^{t}(b(s) / s) d s<\infty, t>0$, because, letting $f(t)=c(t / q), t>0$, we have, for any couple $t, s>0, q t \leqslant s<t$,

$$
(1-s / t) b(t) \leqslant(b(u) / u)((t / q)-(s / q)), \quad s / q \leqslant u \leqslant t / q,
$$


proving our assertion; of course, under the assumptions that $\leqslant$ is the trivial quasi-ordering on $X$ and $b$ is continuous, the particular case just considered yields Altman's Theorem 1.1 stated in [2] (cf. also [5, Theorem 2.1]). It is useful to notice at this moment that an alternative condition with respect to (ix) is

(x) $t \vdash b(t) / t$ is decreasing and $c(t)=\int_{0}^{t}(b(s) / s) d s<\infty, t>0$, showing that our mapping theorem effectively includes that of Altman, because, e.g., the function $b(t)=t^{1 / 2} e^{-t}, t>0$, satisfies (x) but not (ix). A partial extension of these considerations to mappings taking values in a Fréchet space may be found in Turinici [9] (see also [8]). Some useful applications of Theorem 2 (under its particular variants just discussed) to concrete functional equations were indicated by Altman [1].

ACKNOWLEDGEMENT. The author is very indebted to the referee for his valuable suggestions concerning the improvement of the paper.

\section{REFERENCES}

1. M. Altman, Contractors and contractor directions, Theory and Applications, Marcel Dekker, New York, 1977.

2. An existence principle in nonlinear functional analysis, Nonlinear Anal. 2 (1978), 765-771.

3. $\ldots$ A generalization of the Brézis-Browder principle on ordered sets, Nonlinear Anal. 6 (1982), $157-165$.

4. H. Brézis and F. E. Browder, A general principle on ordered sets in nonlinear functional analysis, Adv. in Math. 21 (1976), 355-364.

5. W. J. Cramer and W. O. Ray, Solvability of nonlinear operator equations, Pacific J. Math. 95 (1981), $37-50$

6. I. Ekeland, Nonconvex minimization problems, Bull. Amer. Math. Soc. (N.S.) 1 (1979), 443-474.

7. M. Turinici, A generalization of Brézis-Browder's ordering principle, An. Stiinţ. Univ. “Al. I. Cuza” Iaşi Seçt. I-a Mat. (N.S.) 28 (1982), 11-16.

8. Constant and variable drop theorems on metrizable locally convex spaces, Comment. Math. Univ. Carolinae 23 (1982), 383-398.

9. Mapping theorems via contractor directions in metrizable locally convex spaces, Bull. Acad. Polon. Sci. Sér. Sci. Math. 30 (1982), 161-165.

Seminarul Matematic “Al. MylleR”, University of Iasi, 6600 Iasi, Romania 